\title{
COMPLEXUL PARAZITOID AL CINIPIDULUI NEUROTERUS QUERCUS BACCARUM (HYMENOPTERA, CYNIPIDAE) CE SE DEZVOLTĂ PE FRUNZE DE STEJAR
}

\author{
Manic Gheorghe \\ Rezervaţia Codrii, Strășeni, Lozova, E-mail: manic.gheorghe@gmail.com
}

https://doi.org/10.53937/9789975315975.47

\begin{abstract}
The research of parasitoid calcidids associated with the cinopid Neuroterus quercusbaccarum L. (Hymenoptera, Cynipidae) was performed in the forest ridges of the northern and central districts of the Republic of Moldova during the years 2016-2018. In total, more than 1000 gallons were collected, of which eights pecies of entomophagus included in 7 genres and 6 families were obtained under laboratory conditions: Pteromalidae - Mesopolobus tibialis Westw., M. Fasciiventris Westw.; Eupelmidae - Eupelmus fulvipes Förster, Eulophidae - Olynx gallarum L., Tetrastichus sp., Ormyridae - Ormyrus diffinis Fonsc., Eurytomidae - Eudecatoma biguttata Swed.; Torymidae - Torymus flavipes Walker. In the parasitoid complex with a higher percentage of parasites, threes pecies were found: Torymus flavipes Walker (Chalcidoidea, Torymidae), Mesopolobus tibialis Westw., M. Fasciiventris Westw. (Chalcidoidea, Pteromalidae). The lowest percentage of parasites was recorded in Tetrastichus sp. (Chalcidoidea, Eulophidae). Torymus flavipes Walker iscited as a new species for Moldova's fauna. For the first time there has been infestation of this host with parasitoids: Mesopolobus fasciiventris Westw. (Pteromalidae), Ormyrus diffinis Fonsc. (Ormyridae), Eudecatoma biguttataSwed. (Eurytomidae), Eupelmus fulvipes Förster (Eupelmidae); Tetrastichus sp. (Eulophidae), Torymus flavipes Walker (Torymidae).
\end{abstract}

Key words: parasitoid complex, Neuroterus quercusbaccarum L. (Hymenoptera, Cynipidae).

\section{INTRODUCERE}

Speciile de calcidoide parazitoide asociate cu cinipidul Neuroterus quercus baccarum L. (Hymenoptera, Cynipidae) sunt paraziţi larvari care se dezvoltă în larvele gazdei situate în camera larvară din centrul galei. Complexul parazitoid este format din 8 specii de calcidoide incluse în 6 familii de calcidoide: Pteromalidae - Mesopolobus tibialis Westw., M. Fasciiventris Westw.; Eupelmidae - Eupelmus fulvipes Förster, Eulophidae - Olynx gallarum L., Tetrastichus sp., Ormyridae - Ormyrus diffinis Fonsc., Eurytomidae-Eudecatoma biguttata Swed.; Torymidae - Torymus flavipes Walker . 


\section{MATERIALE ȘI METODE}

Galele formate de insecta galigenă Neuroterus quercusbaccarum L. fixate pe frunze de Quercus robur au fost colectate în formațiunile de pădure cu gorunete situate în zona de nord și codrii centrali ai Moldovei pe parcursul anilor 2016-2018, din care în condiţii de laborator au fost obţinute speciile de calcidoide parazitoide. În total au fost colectate peste 1000 de gale din care au fost obţinute 8 specii de entomofagi incluse în 7 genuri și 6 familii.

\section{REZULTATE ȘI DISCUȚII}

Galele formate de cinipidul Neuroterus quercusbaccarum L. (Hymenoptera, Cynipoidea, Cynipidae), au fost colectate pe teritoriul rezervaţiei „Codrii” pe frunze de Quercus robur. Gala se dezvoltă pe partea inferioară a frunzei, găsindu-se întotdeauna în număr mare de exemplare pe o frunză, deseori câteva zeci și chiar sute (fig. 1).
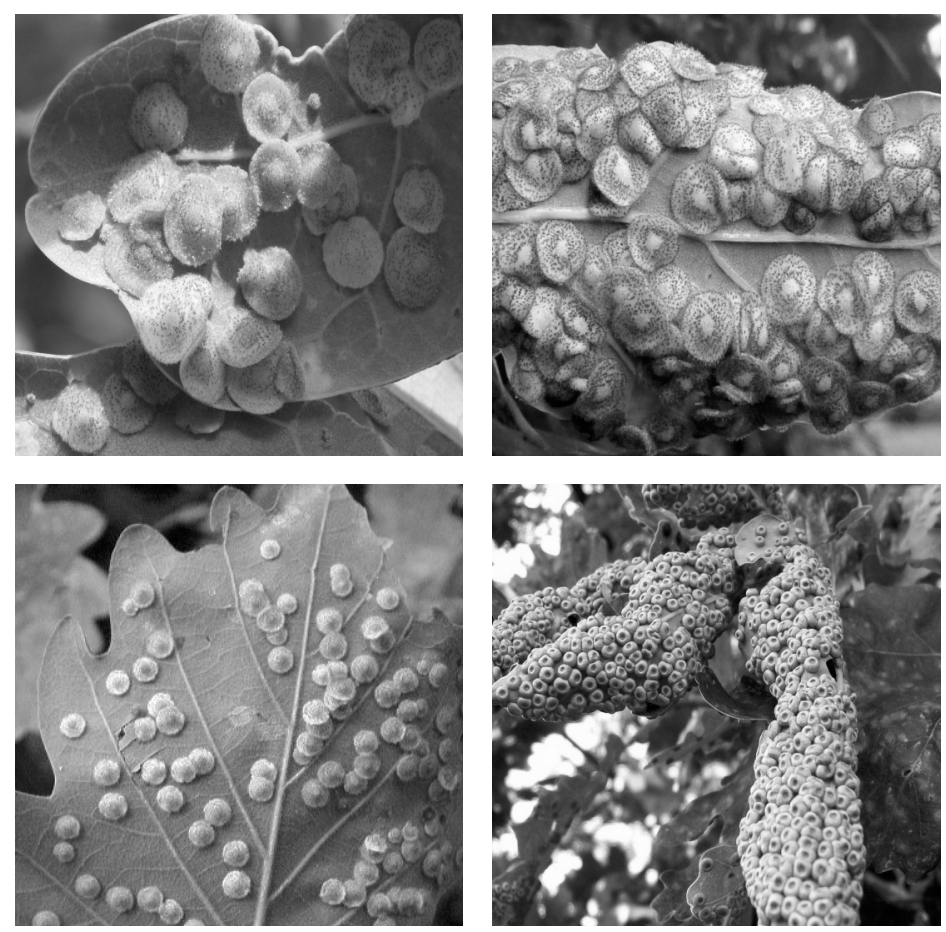

Figura 1. Gale de Neuroterusquercusbacarum pe frunze de stejar

Are forma unui bob de linte (fig. 2 c,d), de 5-6 mm diametru, cu faţa 
superioară convexă, iar cea inferioară plană și cu marginile pe suprafaţa frunzei, fixată într-un singur punct, cu centrul lor. În secţiune o gală areforma unui con foarte obtuz, cu marginile mult subţiate. Pe faţa superioară sunt prezenţi peri scurţi bruni-roșcaţi așezaţi stelat și pe marginea sa cu pete albe, mici, punctiforme. Faţa inferioară este plană și chiar puţin convexă, glabră, iar în mijloc are un peduncul foarte mic și scurt (fig. 2d) cu care se fixează de frunză. Culoarea galelor este galben-brun, la început roșcată. Gala este complet dezvoltată toamna și cade de pe frunză pe pământ în noiembrie, fiind foarte turtită.

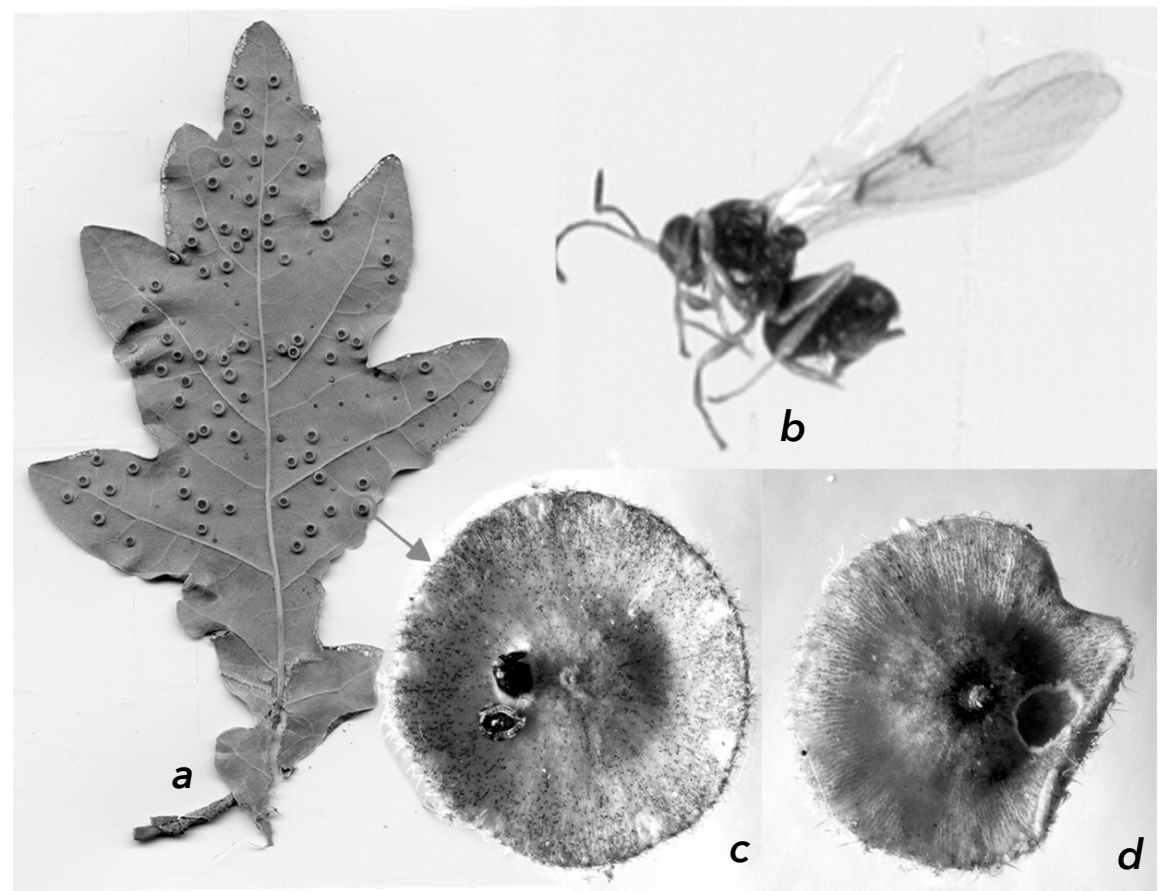

Figura 2. Neuroterus quercus baccarum L.: a- frunză cu gale, b - femelă, c parazitoid, $d$ - orificiu de ieşire

Insectele au corpul negru, fără luciu pronunţat, tegumentul fiind coriaceu, mai mult mat. Caracteristic este aspectul nervurilor aripilor, nervuri groase, brune-negre (fig. 2b), însăși marginea aripilor fiind neagră. Gasterul la mascul este negru, de forma unui bob de linte și pedunculat. Gasterul la femelă este triungiularși sesil. Picioarele la mascul sunt gal- 
bene, cu coxele brune, negre, iar la femelă sunt brune închis, cu unele segmente galbene (fig. 2b). Lungimea corpului la femelă este de 2,5-2,8 $\mathrm{mm}$, la mascul de 2,1-2,8 mm [7].

Au fost obţinute 8 specii de parazitoizi din 6 familii de calcidoide: Pteromalidae -Mesopolobus tibialis Westw., M. Fasciiventris Westw.; Eupelmidae - Eupelmus fulvipes Förster, Eulophidae - Olynx gallarum L., Tetrastichus sp., Ormyridae - Ormyrus diffinis Fonsc., Eurytomidae - Eudecatoma biguttata Swed.; Torymidae - Torymus flavipes Walker.

$\mathrm{Cu}$ un procent mai mare de parazitare s-au evidenţiat calcidoidele: Mesopolobus tibialis Westw., Mesopolobus fasciiventris Westw., Torymus flavipes Walker (fig. 3).

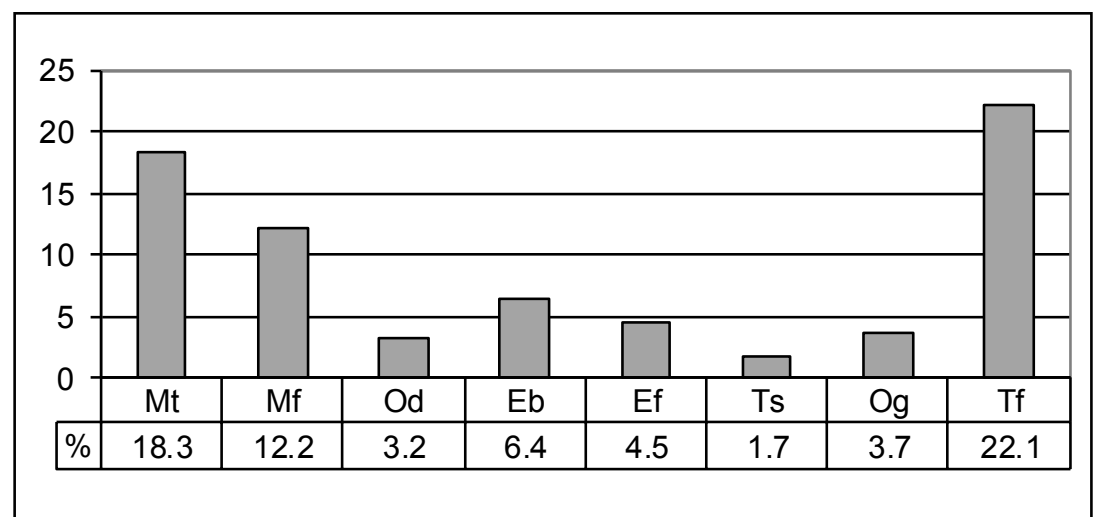

Figura 3. Gradul de parazitare la Neuroterusquercus baccarum (L.)
Mt-MesopolobustibialisWestw
Mf - MesopolobusfasciiventrisWestw
Od - OrmyrusdiffinisFonsc.
Eb -EudecatomabiguttataSwed.

\author{
Ef - EupelmusfulvipesFörster \\ Tsp - Tetrastichus sp. \\ Og - OlynxgallarumLinnaeus \\ Tf -TorymusflavipesWalker
}

Pentru prima dată s-a înregistrat infestarea acestei gazde cu parazitoizii - Mesopolobus fasciiventris Westw., Eudecatoma biguttata Swed,.(4a); Eupelmus fulvipes Förster (4b); Ormyrus diffinis Fonsc. (fig. 4c); Torymus flavipes Walker, Tetrastichus sp. $(4 d, e)[3 ; 5 ; 6]$. 

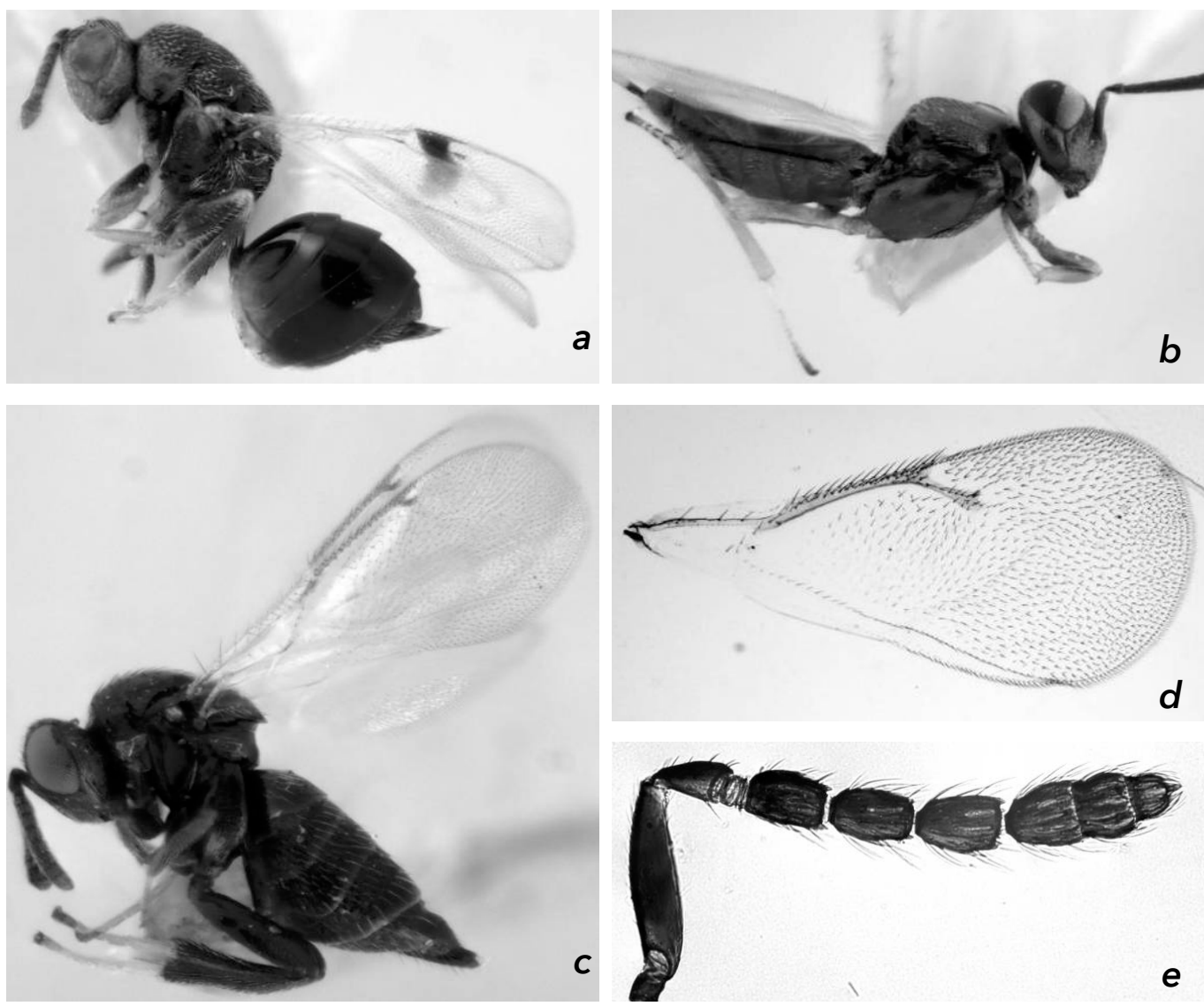

Fig. 4. a - Eudecatoma biguttata Swed $P_{i}$ b - Eupelmus fulvipesFörster $P_{+}$; Ormyrus diffinis Fonsc. ; Tetrastichu ssp. :d - aripă, e - antenă

\section{CONCLUZII}

În complexul parazitoid al cinipidului Neuroterus quercusbaccarum (L.) cu un procent mai mare de parazitare s-au evidenţiat 3 specii: Torymus flavipes Walker (Chalcidoidea, Torymidae), Mesopolobus tibialis Westw., M. Fasciiventris Westw.(Chalcidoidea, Pteromalidae). Cel mai mic procent de parazitare a fost înregistrat la Tetrastichus sp. (Chalcidoidea, Eulophidae). Torymus flavipesWalker este citată ca specie nouă pentru fauna Republicii Moldova. Pentru prima dată s-a înregistrat infestarea acestei gazde cu parazitoizii: Mesopolobus fasciiventris Westw. (Ptero- 
malidae), Ormyrus diffinis Fonsc. (Ormyridae), Eudecatoma biguttata Swed. (Eurytomidae), Eupelmus fulvipes Förster (Eupelmidae); Tetrastichus sp. (Eulophidae), Torymus flavipes Walker (Torymidae) [1; $; 4$ ].

Studiile au fost realizate în cadrul proiectului bilateral AȘM-ASȘIIU, 17.80013.5007.05/Ua.

\section{BIBLIOGRAFIE}

1. Boucek Z. Note s on theChaicid fauna (Chalcidoidea) of Moldavian SSR (in Russianwith English sumary), TrudyMoldavskogonaučno-issledovatel'skogoinstitutasadovodstva i vinodeliâ, Tom VII, Isdatelistvo "Știinţa”, Kišinëv, 1961, p. 5-30.

2. Boucek Z. Materialypo faune hal'cid (Hymenoptera, Chalcidoidea) Moldavskoj SSR, TrudyMoldavskogonaučno-issledovatel'skogoinstitutasadovodstva i vinodeliâ, Tom XIII, Isdatelistvo „Cartea moldovenească”, Kišinëv, 1966, p. 15-38.

3. Boucek Z. \& Raspilus J. Illustrated Keyto West Palearctic Genera of Pteromalidae (Hymenoptera, Chalcidoidea). Techniques et Practiques. INRA. Paris., 1991. 140 pp.

4. Dzhanokmen A. Familly Pteromalida. In: Medvedev, G.S. (Editor in Chiev), - Keys to the Insects of the European Part of USSR (Opredelitel' Nasekomykh Evropeiskoi Chasti SSR. Hymenoptera, 3(2). (Transl. From Russian), rehka printers, New Delhi. India, 1987, p. 88-410.

5. Graham M. The Pteromalidae of North Western Europe(Hymenoptera, Chalcidoidea).Bulletin of the British Museum (Natural History) (Entomology), 1969. Supplement. 16. 1-908 pp.

6. George Melica. Gall waps of Ukraine Cynipidae. KyivVestnik zoologii, 2006. Volume 2, Supliment 21. p. 549-552.

7. Талицкий В., Куслицкий В. Паразитические перепончатокрылые МолАавии. Кишинев, 1990, с.94-110. 